\title{
Inequality, Good Governance and Endemic Corruption
}

\author{
Gil S. Epstein \\ Economics, Bar Ilan University, Ramat Gan \\ IZA, Bonn; GLO; CReAM, London; epsteig@,mail.biu.ac.il \\ Ira N. Gang \\ Economics, Rutgers University \\ RWI, Essen; IOS, Regensburg; IZA, Bonn; GLO; CReAM, London; gang@,rutgers.edu
}

This Version: November 5, 2017

\begin{abstract}
Can a society suffering contests between rich and poor achieve good governance in the face of endemic corruption? We examine a stylized poor state with weak institutions in which a "culture of evasion" damages state authority. Many evade tax payments, limiting the state's economic development capability. In the face of extensive corruption, it is challenging for the state to establish and implement policies reflecting good governance; for example, a government that is accountable and transparent, efficient and effective, and follows the rule of law. The rich and poor possess different views on what is the appropriate level of enforcing proper payments of taxes due. The government needs to design an effective tax administration policy that minimizes corruption and is sensitive to the present and future needs of society. To do this it must understand what drives such widespread corruption.
\end{abstract}

Keywords: corruption, tax administration, governance, rent-seeking

JEL Classifications: O12, O15, 016, D82, G38

Epstein: Department of Economics, Bar-Ilan University, 52900 Ramat-Gan, Israel, epsteig@mail.biu.ac.il, $\quad$ Tel: $\quad+\quad 972 \quad 52 \quad 8 \quad 550 \quad 407, \quad$ Fax: $\quad+972 \quad 3 \quad 5353180$, http://faculty.biu.ac.il/ epsteig.

Gang: Economics Department, Rutgers University, 75 Hamilton St, New Brunswick NJ 089011248 USA, gang@,rutgers.edu, Tel : +1 848-932-8648, Fax : +1 732-932-7416, http://econweb.rutgers.edu/gang/.

(C)2017 Gil S. Epstein and Ira N. Gang. We thank the organizers and participants in WIDER's Public Economics for Development Conference, 5-6 July 2017, Maputo, Mozambique for the opportunity to present an earlier version of this paper and the useful comments provided that helped with this revision. 


\section{Introduction}

Promoting economic development and growth are central roles of government, and we well know that to perform these properly governments must establish the rule of law. While different elements in society may agree on the outlines of what constitutes social welfare, inevitably they disagree on how to achieve it. At some point, self-interest enters the dialogue. Here is where the questions surrounding good governance appear and we ask "Is there a role for good governance in an unequal society faced with endemic corruption?".

What do we mean by endemic corruption? It is corruption that is so ubiquitous that it has become an unremarkable part of life. A bribe, for example, might be required as a normal course of events to continue on your way when stopped by police. Pay-for-play might be so much a part of doing business with the government that it is not thought of as criminal. Using the right accountant to assure you do not face tax evasion accusations might just be what is done. Undoing endemic corruption entails understanding and sensitivity to its context. It cannot simply be declared out of existence.

In capturing endemic corruption, we model tax payment enforcement in an economy with inequality. The government must choose the set of tax administration instruments and effort. We focus primarily on the appropriate level of effort; our conceptualization of the key policy

element in this paper is a single the enforcement level $E$. We discuss and compare the choices facing a government interested in good governance but facing a number of constraints. Our modeling includes a contest between rich and poor members of society trying to influence tax 
collection enforcement for their own benefit (Myles and Naylor, 1996; Schneider and Bose, 2017). The government understands this and reacts to it, reflecting its self-interest and society's. ${ }^{1}$

Our stylized poor state has weak institutions and a "culture of evasion". Many people avoid paying taxes, limiting the state's revenue raising capability and the role it plays in economic development (Andreoni, Erard and Feinstein, 1998). However, the rich and poor want enforcement at different levels. In the face of such extensive corruption, it is challenging to establish and implement policies reflecting good governance.

The next section of the paper sets out the core of our rent-seeking model, in which we examine the workings of a contest in which the rich and poor vie for a tax enforcement plan that serves their own interests. In Section 3, we present the tax administrator (TA), who is characterized as capturing the interests of society as a whole, the government and own selfinterest. Here we explicitly introduce the overlay of TA decision-making into our modeling and examine the model's comparative statics. In Section 4, we discuss several useful extensions of our modeling, including the possibility of a poverty trap the existence and elimination of endemic corruption through the formation of anti-corruption herds. Section 5 concludes.

\footnotetext{
${ }^{1}$ In the seminal work by Yitzhaki (1974) lobbying groups are not taken into consideration.
} 


\section{Tax Payments Enforcement}

We model tax payment enforcement in an economy with inequality. To capture parsimoniously inequality, we posit two agents - one with high income, the other with low income - who take part in a rent-seeking/avoidance contest (Epstein and Nitzan, 1999, 2007). The high/low divide is one among many ways of characterizing the income distribution. Further simplifying the discussion, we refer to high income agents as rich, and those with low income as poor.

The core of our model is the contest between the rich and poor. Both act to maximize their expected net benefit by lobbying the government for their respective optimal levels of tax payment enforcement (Das-Gupta, 2005; Das-Gupta, Ghosh and Mookherjee, 2004). The tax administrator (TA) who proposes and sets the tax enforcement level $E$ represents the government in our model. The TA is imperfectly honest, both wanting to receive rents from the efforts of the rich and poor to influence her/his decisions and wanting to act in the best interests of the country by reflecting the prevailing sentiment in the society with respect to tax avoidance and enforcement (Flatters and MacLeod, 1995). Thus, rent-seeking/avoidance is an important part of the corruption story.

Subsections 2.1 and 2.2 formally establish what the contest between the rich and poor looks like, given that both of these actors are trying to influence TA actions.

\subsection{The Contest between Rich and Poor}

A tax administrator (TA) establishes the contest by proposing tax enforcement level $E$ and a contest success function (CSF). The CSF converts rent-seeking/avoidance efforts by the rich and poor to influence the TA's proposed enforcement level $E$ into probabilities of approval and rejection. With this information the rich and the poor decide on their lobbying expenditures (that 
is, their strategies), allowing us to obtain the enforcement level assuming the contest has a Nashequilibrium and complete information on the parameters is available. The equilibrium outcome from the rent-seeking/avoidance contest is the TA's political constraint. The policy the TA follows reflects its various commitments and its political constraint.

Before proceeding further, we should spend a minute discussing our conceptualization of the key policy element in this paper, the enforcement level E. Clearly, many elements of tax administration policy need consideration in actual practice, and using the single policy $E$ is overly simple. However, it does focus our story. Das-Gupta, Estrada and Park (2016) have recently offered the empirical analogue of this. They propose and construct a Tax Administration Measure of Effectiveness (TAME), an index that captures the tax administration environment including its effectiveness and enforcement. ${ }^{2}$ We rely on this conceptualization.

The risk-neutral poor $(p)$ and rich $(r)$ spend $x_{p}$ and $x_{r}$ on rent-seeking/avoidance. Expenditure by the rich and poor in this lobbying contest correlates closely to their chances of winning. Expenditures $x_{p}$ and $x_{r}$ establish the approval probability of the TA's proposed policy E; e.g., with probability $\operatorname{Pr}_{p}$ the TA accepts the poor's proposed enforcement level E. For convenience, we set the enforcement level desired by the rich at zero so that the rich are not the subjects of tax enforcement. In general, we can think of this as setting their $E$ at some minimum level enabling the rich to obtain their desired level of public goods.

Enforcement level $E^{*}$ maximizes the payoff to the poor. Whereas the rich prefer minimal enforcement, the poor's preferred enforcement level is positive and high enough to ensure the

2 Das-Gupta, Estrada and Park (2016) suggest that TAME is composed of numerous factors. Summarizing, these include: TA ability to audit, inspect, penalize, prosecute; number and quality of tax inspectors, information, balance sheets, TA budget; efficient resource allocation such as taxpayer identification, and registration, processing of returns, audits, post-audit appeals, sanctions, tax collection, taxpayer assistance, internal audits; output per inspector; duration, arrears, revenue loss duration of assessments completion, appeals, etc., and quality. 
availability of a sufficient level of public goods, but not too high, as this may hurt them. The poor understand the benefit of paying taxes as aiding public goods provision while recognizing that the cost of paying too much also needs consideration.

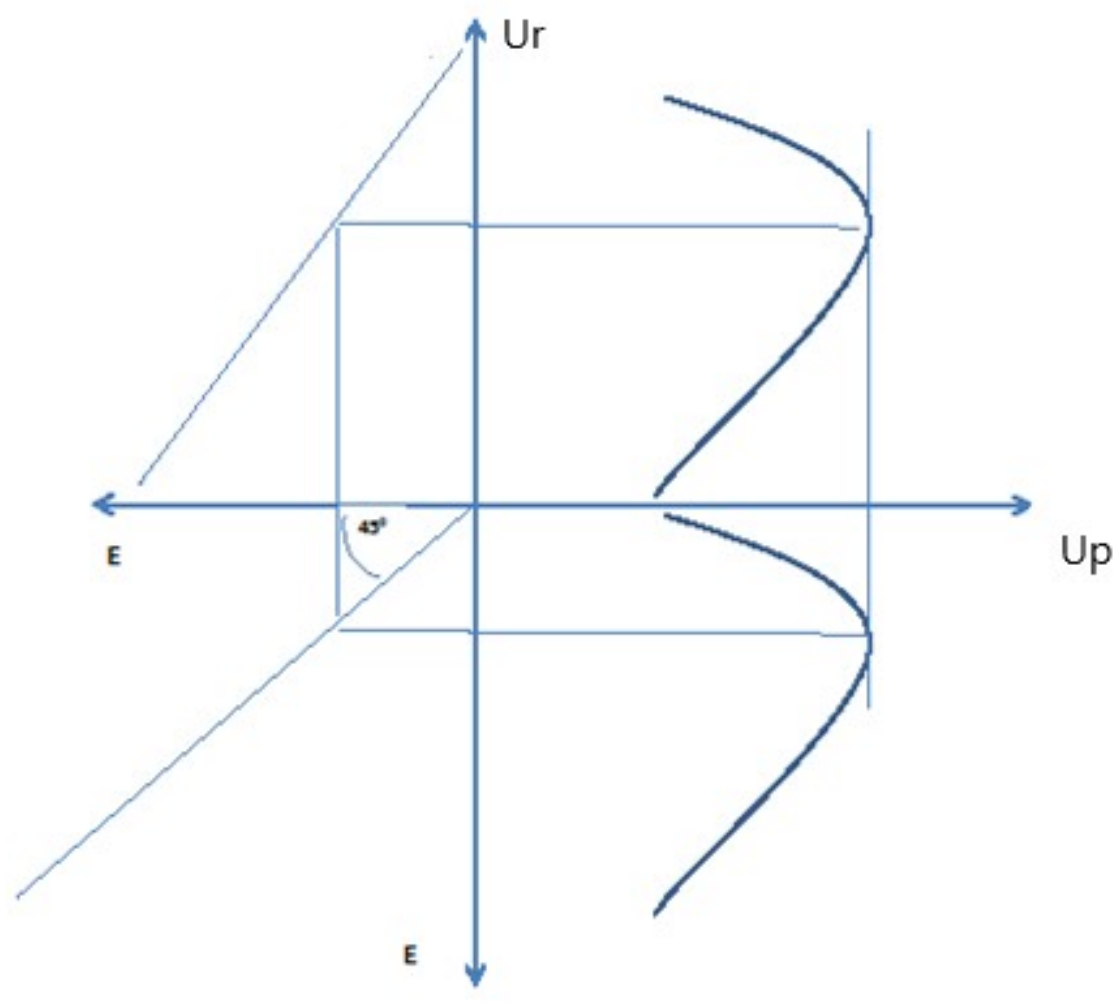

Figure 1: Basic Model Relationships

Even before specifying equations, we can examine the relationships heuristically in Figure 1, where $E$ is tax administration enforcement (for example, the TAME index) and $u_{r}$ and $u_{p}$ are the welfare of the rich and poor, respectively. The relationship between enforcement and the preferences of the rich is illustrated in quadrant II as linear, with utility increasing as enforcement decreases. For the poor the relationship depicted in quadrant IV is a non-linear "inverted-U", with utility rising, peaking and falling as enforcement increases. The rich are better off with very low enforcement; the poor with some in-between level. We see the 
relationship between how well off the rich and poor are with regard to one another in quadrant I.

The rich and the poor have expected net payoffs (surpluses) of ${ }^{3}$

$$
E\left(u_{r}\right)=A_{r}+\operatorname{Pr}_{r} n_{r}-x_{r},
$$

and,

$$
\boldsymbol{E}\left(u_{p}\right)=A_{p}+\operatorname{Pr}_{p} n_{p}-x_{p} .
$$

$E\left(u_{r}\right)$ and $E\left(u_{p}\right)$ are expected net payoffs to the rich and poor, respectively. $A_{r}$ and $A_{p}$ are the respective payoffs of the rich and poor; $n_{r}$ and $n_{p}$ are the real benefits each group receives from their rent-seeking/avoidance activities. The sizes of the benefits going to each group are a function of the enforcement level. $\operatorname{Pr}_{r}$ and $\operatorname{Pr}_{p}$ are the contestants' probabilities of winning the contest (note, $P r_{r}+P r_{p}=1$ ). $\quad x_{r}$ and $x_{p}$ are their contest expenditures. The stakes, the $n$, are a function of the enforcement level; in equilibrium, so the probabilities.

If the TA rejects the proposed enforcement level, the rich receive real benefit $n_{r}$; in fact, a win for the rich provides them with an avoided loss. Victory belongs to the poor if the TA approves the proposed enforcement level, as this is their preferred outcome, and they receive $n_{p}$.

The rich receive payoff (profits) $A_{r}$ when the TA does not adopt their favored enforcement level; $n_{r}$ is the winning benefit received by the rich in the contest. Note again that the enforcement level is set at zero when the rich win the contest. The poor's payoff is $A_{p}$ when the TA does not accept their favored policy; $n_{p}$ is the winning benefit received by the poor. Therefore, if the poor win the contest they get $A_{p}+n_{p}$ otherwise they get $A_{p}$.

From the CSF we obtain probabilities $P r_{r}$ and $P r_{p}$; i.e., the CSF converts expenditures by the rich and poor into the probabilities they can obtain their favored policy outcome. We assume the

\footnotetext{
${ }^{3}$ For a similar structure see Epstein and Nitzan $(2003,2007)$
} 
TA chooses Tullock's (1980) non-discriminating rule that player i's success probability when competing against player $j$ is

$$
\operatorname{Pr}_{i}=\operatorname{Pr}_{i}\left(x_{i}, x_{j}\right)=\frac{x_{i}}{x_{i}+x_{j}}, \forall i \neq j, \quad x_{i}, x_{j}>0
$$

The interior Nash equilibria of our two-player contest (subgame) is characterized by

$$
\frac{\partial \boldsymbol{E}\left(u_{r}\right)}{\partial x_{r}}=\frac{x_{p}}{\left(x_{r}+x_{p}\right)^{2}} n_{r}-1=0 \text {, and } \frac{\partial E\left(u_{p}\right)}{\partial x_{p}}=\frac{x_{r}}{\left(x_{r}+x_{p}\right)^{2}} n_{p}-1=0 \text {. }
$$

The second order sufficient conditions of such equilibria are satisfied.

Rich and poor equilibrium expenditures and winning probabilities are

$$
x_{r}^{*}=\frac{n_{r}^{2} n_{p}}{\left(n_{r}+n_{p}\right)^{2}} ; x_{p}^{*}=\frac{n_{p}^{2} n_{r}}{\left(n_{p}+n_{r}\right)^{2}} ; \operatorname{Pr}_{r}^{*}=\frac{n_{r}}{n_{r}+n_{p}} ; \operatorname{Pr}_{p}^{*}=\frac{n_{p}}{n_{r}+n_{p}}
$$

From (5), we see that whoever has the higher benefit also has the higher probability of winning the contest.

\subsection{Relative Efforts, Winning Probabilities and the Proposed Enforcement Level}

We now consider some of the consequences of the model set up in Section 2.1; we examine here the comparative statics of the contest. Proposed enforcement level $E$ changes on equilibrium expenditures by the rich and poor are

$$
\frac{\partial x_{r}^{*}}{\partial E}=n_{r} n_{p} n_{r}^{\prime}\left(n_{r}-n_{p}\right)\left[\frac{\eta_{p}}{\eta_{r}}+2 \frac{n_{p}}{n_{r}-n_{p}}\right] \frac{1}{\left(n_{r}(E)+n_{p}(E)\right)^{3}},
$$

$$
\text { and }
$$

$$
\frac{\partial x_{p}^{*}}{\partial E}=n_{r} n_{p} n_{p}^{\prime}\left(n_{p}-n_{r}\right)\left[\frac{\eta_{r}}{\eta_{p}}+2 \frac{n_{r}}{n_{p}-n_{r}}\right] \frac{1}{\left(n_{r}(E)+n_{p}(E)\right)^{3}},
$$


where $\eta_{j}=\frac{\partial n_{j}}{\partial E} \frac{E}{n_{j}}=n^{\prime}{ }_{j} \frac{E}{n_{j}}$ is contestant $j$ 's benefit elasticity with respect to changing the proposed enforcement level E.

For $E^{*}>E>0$ notice that $\frac{\partial n_{j}}{\partial E}=n^{\prime}{ }_{j}>0, \forall j=p, r$ and for $E>E^{*}$, $\frac{\partial n_{p}}{\partial E}<0$ and $\frac{\partial n_{r}}{\partial E}>0$. Also notice that there exists $\bar{E}$, such that the poor prefer an enforcement level $E$ exceeding the optimal level $E^{*}\left(E^{*}<E<\bar{E}\right)$ rather than a zero enforcement level. Then again, a very high enforcement level $E,(E>\bar{E})$, is worse than a zero enforcement level .

Thus, the effect of changes in enforcement on outlays made by the rich and poor is ambiguous. The critical elements here are the contestants' benefit elasticities with respect to changes in the enforcement level.

By (5),

$$
\frac{\operatorname{Pr}_{r}^{*}}{\operatorname{Pr}_{p}^{*}}=\frac{x_{r}^{*}}{x_{p}^{*}}=\frac{n_{r}(E)}{n_{p}(E)}
$$

Since,

$$
\frac{\partial\left(\operatorname{Pr}_{r}^{*} / \operatorname{Pr}_{p}^{*}\right)}{\partial E}=\frac{\partial\left(x_{r}^{*} / x_{p}^{*}\right)}{\partial E}=\frac{n_{r}(E)}{E n_{p}(E)}\left(\eta_{r}-\eta_{p}\right) .
$$

We obtain, 


\section{Proposition 1:}

a. If $E^{*}>E>0$, a proposed enforcement level $E$ change on the odds of the rich will win the contest and their relative contest expenditure is ambiguous. Elasticities $\eta_{r}$ and $\eta_{p}$ drive this effect. Specifically,

$$
\frac{\partial\left(\operatorname{Pr}_{r}^{*} / \operatorname{Pr}_{p}^{*}\right)}{\partial E}=\frac{\partial\left(x_{r}^{*} / x_{p}^{*}\right)}{\partial E}<0 \text { if } \quad \eta_{r}-\eta_{p}<0 .
$$

b. If $\bar{E}>E>E^{*}$, a proposed enforcement level $E$ change affects directly the odds the rich will win the contest and their relative contest expenditure. Namely,

$$
\frac{\partial\left(\operatorname{Pr}_{r}^{*} / \operatorname{Pr}_{p}^{*}\right)}{\partial E}=\frac{\partial\left(x_{r}^{*} / x_{p}^{*}\right)}{\partial E}>0
$$

This proposition directly characterizes the chances each player has of winning the contest when there is a change in the proposed enforcement level, where winning the contest means obtaining its' desired enforcement level from the TA. The relationship between elasticities $\eta_{r}$ and $\eta_{p}$ plays a key role in case (1.a). That is, with respect to changing the proposed enforcement level $E$, how elastic is the rich's benefit compared to the poor's benefit. When $\eta_{r}$ exceeds $\eta_{p}$, the poor have a better chance of winning and their relative rent-seeking efforts increase. However, in case (1.b) the poor's benefit decreases while that of the rich increase with proposed enforcement level increases. Therefore, the poor are more likely to lose and they decrease their relative rentseeking efforts. 
Let us now examine what happens to the sum of expenditures by the rich and poor on achieving their enforcement goals. Where $X$ is total rent-seeking/avoidance expenditures (or effort, also referred to as rent-dissipation), by (5), in equilibrium we have

$$
X^{*}=x_{r}^{*}+x_{p}^{*}=\frac{n_{r}(E) n_{p}(E)}{n_{p}(E)+n_{r}(E)}
$$

We are interested in how the sum of expenditures by the rich and poor as they try to influence the contest is related to the proposed enforcement level $E$,

$$
\frac{\partial X^{*}}{\partial E}=\frac{n_{p}(E) n_{r}(E) n_{p}{ }_{p}(E)}{\left(n_{r}(E)+n_{p}(E)\right)^{2}}\left(\frac{\eta_{r}}{\eta_{p}}+\frac{n_{r}(E)}{n_{p}(E)}\right) \text {. }
$$

That is, generally, it is ambiguous. More specifically, we can characterize when overall rentdissipation is increasing by

\section{Lemma 1:}

a. If $E^{*}>E>0$, then $\frac{\partial X^{*}}{\partial E}>0$.

b. If $\bar{E}>E>E^{*}$, then $\frac{\partial X^{*}<}{\partial E}>0$ if $\frac{-\eta_{r}}{\eta_{p}}>\frac{n_{r}}{n_{p}}$.

Interestingly, under the sufficient condition, $-\eta_{r}<\eta_{p}$, the sum of rent-seeking expenditures by the rich and poor are inversely related to the proposed enforcement level, E. This lemma characterizes rent-dissipation.

What happens to each player's expenditures when the proposed enforcement level changes? (1.a) tells us when total expenditure (that is, total effort or rent-dissipation) increases when proposed enforcement increases if $E$ is below the poor's optimal enforcement level, $E^{*}$. The 
contest between the rich and the poor becomes more intense as the respective benefits are high. Even when an increase persuades the poor to reduce their rent-seeking activities, the rich counterbalance this reduction by disproportionately increasing their rent-avoidance efforts. (1.b) stipulates the circumstances that tell us how equilibrium total rent-seeking/avoidance expenditures react to proposed enforcement level changes, when $E$ is above the poor's optimal enforcement level, $E^{*}$.

\section{Tax Administration}

We characterized in Section 2 the contest between the rich and the poor. Here we consider social welfare, explicitly accounting for the actions of the tax administrator (TA), their consequences, and the responses of the rich and the poor. The TA takes the rentseeking/avoidance contest discussed in Section 2 as a political constraint. Commitment to the populace and self-interest motivate the TA.

\subsection{The Politically Constrained Tax Administrator}

The tax administrator (TA) establishes the enforcement level $E$ taking into account the contest between the rich and the poor, its desire to acquire rents and its commitment to enhancing social welfare. The TA's specific objective function captures these commitments. The TA gains from expenditures made to lobby with regard to the proposed enforcement level and from the four possible payoffs to the rich and the poor, because part or all of their expenditures are a resource

transfer to the TA. Hence, the objective function is $G\left(\boldsymbol{E}\left(u_{r}\right) ; \boldsymbol{E}\left(u_{p}\right) ;\left(x_{r}+x_{p}\right)\right)$ for the TA. The expected net payoffs to the rich and poor are $E\left(u_{r}\right)$ and $E\left(u_{p}\right)$ as per equations (1) and (2). The contestants' expenditures $\left(x_{r}+x_{p}\right)$ either are wasted lobbying resources or represent 
transfers to the government (of which the TA is the agent). The TA gains the greater the fraction of the expenditures it captures and the sum of rich and poor expected payoffs to others.

Player l's equilibrium expected payoff, $l=$ rich or $l=$ poor, is $\bar{E}\left(u_{l}^{*}\right)$; i.e., $\overline{\boldsymbol{E}}\left(u_{l}^{*}\right)$ is player l's equilibrium expected payoff disregarding rent-seeking/avoidance costs, $E\left(u_{l}^{*}\right)=\overline{\boldsymbol{E}}\left(u_{l}^{*}\right)-x_{l}^{*}$. Assume an additive TA objective function,

$$
G\left(\boldsymbol{E}\left(u_{r}\right) ; \boldsymbol{E}\left(u_{p}\right) ;\left(x_{r}+x_{p}\right)\right)=\alpha\left(\overline{\boldsymbol{E}}\left(u_{p}^{*}\right)+\bar{E}\left(u_{r}^{*}\right)\right)+(1-2 \alpha)\left(x_{p}^{*}+x_{r}^{*}\right) .
$$

The TA has mixed commitments. The weight $(1-2 \alpha)$ determines whether $\left(x_{p}{ }^{*}+x_{r}{ }^{*}\right)$ adds to or subtracts from welfare. If the total rent-seeking/avoidance expenditures are wasted, the weight is negative. Weights $\alpha$ and $1-2 \alpha$ apportion the welfare components, with $\alpha$ capturing the TA's mixed commitments to the public and self. This parameter reflects the prevailing sentiment in the society with respect to tax avoidance and enforcement, echoing contestants' expenditure allocations between wasteful and non-wasteful resources received by the TA. Here we see the TA's public commitment and narrow self-interest in collecting contestants' expenditures.

Given (11), when $\alpha=1$, the TA is totally committed to the public interest and society views expenditures on rent-seeking/avoidance as completely wasteful. When $\alpha=1 / 2$ and we again observe a fully committed TA, expenditures made by the rich and the poor are in fact a transfer from the rich and poor to the government that reallocates it back to them. In the extreme case, $\alpha=0$. Here the TA's objective is maximizing rich and poor expenditures on the contest while ignoring the public's welfare. The TA cares only about getting contest rent! Other intermediate cases are obtained for $0<\alpha<1, \quad \alpha \neq 1 / 3, \quad \alpha \neq 1 / 2$. When $0<\alpha<0.5$, efforts at rentseeking/avoidance positively affect the TA; when $0.5<\alpha<1$, the effect on the TA's objective function is negative. 


\subsection{The Equilibrium Enforcement level}

To determine the optimal level of E maximizing the TA's objective function (11), we look at the interior solution to the TA's problem, characterized by the first order condition

$$
\frac{\partial G(.)}{\partial E}=\alpha \frac{\partial\left(\bar{E}\left(u_{r}^{*}\right)+\bar{E}\left(u_{p}^{*}\right)\right)}{\partial E}+(1-2 \alpha) \frac{\partial X^{*}}{\partial E}=0
$$

or,

$$
\frac{\partial\left(\overline{\boldsymbol{E}}\left(u_{r}^{*}\right)+\overline{\boldsymbol{E}}\left(u_{p}^{*}\right)\right)}{\partial E}=-\frac{(1-2 \alpha)}{\alpha} \frac{\partial X^{*}}{\partial E}
$$

Assuming the order second condition holds, $\frac{\partial^{2} G(.)}{\partial E^{2}}=\alpha \frac{\partial^{2}\left(\bar{E}\left(u_{r}^{*}\right)+\bar{E}\left(u_{p}^{*}\right)\right)}{\partial E^{2}}+(1-2 \alpha) \frac{\partial^{2} X^{*}}{\partial E^{2}}<0$. (4) and (14) characterize an interior Stackelberg-Nash equilibrium $\left(x_{r}^{*}, x_{p}^{*}, E^{* *}\right)$. This term and especially $\mathrm{E}^{* *}$ pops up from nowhere.

Turning to examine how changes in $\alpha$, the parameter summarizing the sensitivity of the society and the TA to corruption and evasion, affect the equilibrium enforcement level $E^{*}$, it can be shown that $\frac{\partial E^{* *}}{\partial \alpha}=\frac{-\partial^{2} G(.) / \partial E \partial \alpha}{\partial^{2} G(.) / \partial E^{2}}$. By the second order condition, $\frac{\partial^{2} G(.)}{\partial E^{2}}<0$. By means of the first order conditions, we can see that $\frac{\partial E^{* *}}{\partial \alpha}$ and $\frac{\partial X^{*}}{\partial E}$ have opposite signs. Hence, 


\section{Proposition 2:}

$$
\operatorname{Sign}\left(\frac{\partial E^{* *}}{\partial \alpha}\right)=-\operatorname{Sign}\left(\frac{\partial X^{*}}{\partial E}\right)
$$

Via lemma 1, we immediately see the conditions determining the sign of $\left(\frac{\partial E^{* *}}{\partial \alpha}\right)$.

$E^{* *}$ is the equilibrium enforcement level in our extended tax enforcement game. However, the poor prefer the level maximizing their expected net payoff, $E^{*}$. We can compare $E^{*}$ and $E^{* *}$ by examining the relationship between the TA's/society's sensitivity to corruption $(\alpha)$ and this comparison's outcome. We want to know: (i) Is there an $\alpha$ which gives rise to an enforcement level $E^{* *}$ equal to $E^{*}$ ? (ii) Is there an $\alpha$ which gives rise to an enforcement level $E^{* *}$ equal to the level the rich prefer (which we denote by zero)?

When $\alpha=0$, the TA's objective is maximizing rent-seeking/avoidance expenditures while ignoring public welfare. Such an assumption implies a TA who is totally committed to his own narrow interest in governing the expenditures made by the rich and poor who are each trying to win the contest. The equilibrium enforcement level $E^{* *}$ maximizes $G(E)=X^{*}(E)$ and satisfies the first order condition

$$
\frac{\partial X^{*}}{\partial E}=0
$$

By lemma 1, it is clear that if $\frac{-\eta_{r}}{\eta_{p}}<\frac{n_{r}(E)}{n_{p}(E)}$, then $E^{* *}=E^{*}$, otherwise $E^{* *}>E^{*}$. We thus obtain 


\section{Proposition 3:}

a. There exists $\alpha_{1}$, such that $E^{* *}\left(\alpha_{1}\right)=E^{*}$.

b. If $\frac{-\eta_{r}}{\eta_{p}}<\frac{n_{r}(E)}{n_{p}(E)}$, there exists $\alpha_{2}$ such that $E^{* *}\left(\alpha_{2}\right)>E^{*}$.

Proposition 3 tells us that this society possesses a sensitivity to corruption that brings about the enforcement level $\mathrm{E}^{*}$ that is preferred by the poor. The proposition also informs us about conditions ensuring the proposed enforcement level exceeds $E^{*}$. The introduction of the enforcement level $\mathrm{E}^{*}$ in our setting may require positive $\alpha$, i.e., that the TA assigns a positive weight to the public's aggregate expected benefit.

We argued above that if the poor win the contest with certainty, they prefer the TA set the enforcement level at $E^{*}$, maximizing the poor's benefit $n_{p}$, and satisfying: $\left.\frac{\partial n_{p}(E)}{\partial E}\right|_{E=E^{*}}=0 . E^{*}$ is the optimal level of enforcement maximizing the poor's benefit. It does not maximize the poor's expected benefit. Thus it only looks at the benefits and does not take into consideration the probability of winning or losing the contest or the level of expenditure invested in the contest. However, the poor do not win the contest with certainty in our extended strategic setting, so they need to take into consideration the possibility that increasing their net benefit (by increasing E), may reduce their probability of winning the contest by increasing opposition by the rich. The poor in our setting prefer the TA to set the enforcement level at $E_{0}$ - the enforcement level maximizing their equilibrium expected net payoff. From (2) and (6), the poor's expected payoff is 


$$
\boldsymbol{E}\left(u_{p}^{*}\right)=A_{p}+\frac{\left(n_{p}(E)\right)^{3}}{\left(n_{r}(E)+n_{p}(E)\right)^{2}} .
$$

The positive enforcement level $E=E_{0}$ maximizing $E\left(u_{p}^{*}\right)$ is characterized by the first order condition:

$$
\frac{\partial \boldsymbol{E}\left(u_{p}^{*}\right)}{\partial E}=\frac{3\left(n_{p}(E)\right)^{2} \frac{\partial n_{p}(E)}{\partial E}}{\left(n_{r}(E)+n_{p}(E)\right)^{2}}-\frac{2\left(n_{p}(E)\right)^{3}\left(\frac{\partial n_{p}(E)}{\partial E}+\frac{\partial n_{r}(E)}{\partial E}\right)}{\left(n_{r}(E)+n_{p}(E)\right)^{3}}=0 .
$$

At $E^{* *}=E^{*}, \quad \frac{\partial n_{p}(E)}{\partial E}=0$. Since, $\frac{\partial n_{r}(E)}{\partial E}>0$ at $E^{*},\left.\frac{\partial \boldsymbol{E}\left(u_{p}^{*}\right)}{\partial E}\right|_{E=E^{*}}<0$. This implies that the enforcement level maximizing the poor's expected net payoff is smaller than $E^{*}$. Summing up:

\section{Proposition 4:}

(a) $E_{0}<E^{*}$.

(b) $\alpha_{3}$ exists, such that $E^{*}\left(\alpha_{3}\right)=E_{0}$.

The level of enforcement that maximizes the poor's payoff is $E^{*}$. However, if they also take into consideration the probability of winning and the level of expenditure needed to try to win approval (in equilibrium the probabilities, the benefits and the expenditure are all functions of the level of enforcement) we will obtain that the optimal enforcement level maximizing the expected payoff is lower that what maximizes the payoff: $E_{0}<E^{*}$. The reason is that if they ask 
for a lower level, the resistance of the rich will be lower and the poor may have a higher probability of winning.

Given the expected payoff of both groups, the TA maximizes his expected payoff as staged in (11). For each given $\alpha$ (the weight the TA assigns to welfare), the level of enforcement that the TA proposes will be different. Thus the proposition states that there exists an $\alpha$ such that the enforcement level that the TA proposes will be identical to the level that the poor prefer. If the TA operates at a level where corruption sensitivity is embodied by $\alpha_{3}>0$, we are describing a TA who is more committed to enhancing social welfare than a TA whose equilibrium policy equals $E^{*}$.

\section{The Implications of the Poor's Constraint}

In this section, we offer several useful considerations of our modeling: (1) how hard the rich and poor fight for their desired tax enforcement levels; (2) the possibility of herding by taxpayers; (3) change in the TA's/society's sensitivity to corruption; (4) a poverty trap. Up to here we have observed that the proposed optimal enforcement level may well be even higher than what the poor would like; however, it may also be lower than the poor's desires (since they receive benefits from collected taxes) and closer to the wishes of the rich (which is zero enforcement). This depends on the sensitivity of the political culture to corruption and reduced tax enforcement.

Recall we showed the optimal level of enforcement maximizing the benefit to the poor is $E^{*}$, while the level maximizing the expected net payoff of the poor is $E_{0}$ and it holds that $E_{0}<E^{*}$. Assume that the proposed enforcement level is set at $E_{0}$. From (5) the expenditure of 
the poor attempting to influence the proposed policy equals $x_{p}^{*}\left(E_{0}\right)=\frac{n_{p}{ }^{2}\left(E_{0}\right) n_{r}\left(E_{0}\right)}{\left(n_{p}\left(E_{0}\right)+n_{r}\left(E_{0}\right)\right)^{2}}$. As the desired enforcement level the rich prefer is lower than that of the poor, the level of expenditure under $E_{0}$ is more than what is optimal for the rich.

The expected net payoff to the poor would be: $\boldsymbol{E}\left(u_{p}^{*}\right)=A_{p}+\frac{\left(n_{p}\left(E_{0}\right)\right)^{3}}{\left(n_{r}\left(E_{0}\right)+n_{p}\left(E_{0}\right)\right)^{2}}$, which is higher than if the enforcement level was equal to zero (the desired level for the rich). However, we have to remember that the benefit is in expected terms while the expenditure is absolute. This means that the revealed payoff may be negative since expenditure may be too high. The outcome may be either

$$
u_{p}=A_{p}-x_{p}(E)
$$

if they lose the competition, or if they win

$$
u_{p}=A_{p}+n_{p}(E)-x_{p}(E) .
$$

If it holds that $u_{p}=A_{p}-x_{p}(E)<0$, then the poor may decide not to invest resources or will invest fewer resources than needed to attain the optimal $E$ as (18) is negative and they will invest such that (18) will be positive - they will invest less than is necessary to maximize their expected net payoff. This gives the rich more influence in determining (together with the TA) the enforcement level. In other words, even though the poor should fight to increase enforcement, they may well not do so. ${ }^{4}$

\footnotetext{
${ }^{4}$ Instead of the payment being less than zero as in equation (18), we could write it as less than some positive threshold. See the discussion on the poverty trap below.
} 
In the case just presented the rich will have a greater influence on determining the country's enforcement measures. Hence, lower enforcement is generally the rule. The poor can obtain more from the government then they pay in taxes. The reason is simply that their income is low and the benefits they get from government are higher than what they pay in. On the other hand, they do not have many resources and it is not clear that they can afford to invest resources in attempting to affect the enforcement level. Notice that poor, low-income individuals wish tax enforcement to be stricter than desired by the rich. As the poor pay less to the government then they receive in benefits from the government, their net tax payment is negative - they want taxes to be properly collected.

What can change this? Consider herding by taxpayers and the TA's/society's sensitivity to corruption

Herd effects: Slowly, very slowly, people will stop evading taxes, as they will see others not evading. With heterogeneous people first those who are most affected - most sensitive, those with a lower threshold - will stop evading (Epstein and Gang, 2010), This will increase the actual level of enforcement observed by others and will increase the benefits the poor obtain, enabling (18) to be positive. What this means is that the poor will be able to increase their efforts to have increased enforcement, eventually drawing in those with a higher threshold level and slowly further increasing the number of not evading. Finally, it will affect the rich. What we are observing here is essentially 'backwards herding'; i.e., instead of evasion leading to increased evasion as people described in Epstein and Gang (2010), enforcement acts as to generate increased honesty which further increases honesty potentially until there is no longer any tax evasion. 
A change in the TA's/society's sensitivity to corruption: Here too, we might observe 'backwards herding'. If the TA/society becomes more sensitive to corruption - that is, $\alpha$ in our model increases - this would reduce the influence of the rich on the chosen level of tax enforcement. It seems that the higher possibility would be the first possibility, thus a herd affect will start paying taxes that will start affecting all others. Like the herd that started not paying taxes, in the same way, a herd can start to pay taxes that will affect the rest of the population.

Finally, consider the possibility of a poverty trap. Instead of a less than zero outcome holding as we have just discussed for equation (18), think of the outcome as less than some threshold. The poor will not now invest in trying to change the level of payment enforcement that is they will not make these types of expenditures. Thus, the enforcement level decreases. In a heterogeneous population, first those with low payoffs $A$ stop investing possibly affecting those with higher payoffs such as $A r$ which will then cause others to not invest, making the poor poorer and poorer. Thus, we have a trap!

\section{Conclusion}

Tax administration policy is the set of instruments by which the government influences voluntary and involuntary compliance in trying to achieve its goals. Our goal is to understand some of the forces involved in determining tax administration policy by examining the contest over it. Many papers on tax administration and compliance address their tax revenue impact in an accounting sense and under assumptions of the standard economic models of tax evasion (Das-Gupta and Gang, 2000). Within a theoretical model, we discuss and compare choices facing the government over tax administration and behavioral instruments, where the 
government faces large-scale endemic corruption and wants to achieve the highest aggregate level of good governance.

Frequently economic policy results from a contest among concerned parties who argue for their desired policy and against the suggestions of others. In our story the rich and poor are engaged in a struggle determining the degree of tax enforcement. The struggle can take different forms including bribery, lobbying, rent-seeking, protests. The outcome depends on the payoffs the contestants receive if the tax administrator chooses their desired enforcement level, or not.

Efforts of Transparency International, the World Bank and others who evaluate institutions have raised awareness of the costs of doing business and living in different environments. We build a story here of how awareness of corruption affects tax enforcement. The story is not always straightforward. We characterize an economy in which there is inequality and tax evasion by both rich and poor, but these two groups have different interests and therefore use their resources trying to influence the efforts made to enforce tax payments at the level that benefits them the most. The government's multiple objectives are captured here in the character of the tax administrator, who we picture a multifaceted individual facing personal conflicts captured in a welfare function.

The government needs to design an effective tax administration policy that minimizes corruption and is sensitive to society's present and future needs. The tax administrator (TA) acting as the government's agent faces choices in searching for the mechanism that will achieve the highest aggregate level of good governance over the set of tax administration instruments and efforts.

We examine a stylized poor state with weak institutions in which a "culture of evasion" damages state authority. Many people evade tax payments, limiting the role the state can play in 
economic development. In the face of such extensive corruption, it is challenging to establish and implement policies reflecting good governance, i.e., maximizing social welfare.

We lay out the lobbying contest between the rich and poor, in which the tax authority acts on behalf of social welfare and its own self-interest to determine the tax enforcement level, while understanding the contest that is going on. The effect of the enforcement level on the outlays of the rich and poor hinges on the elasticities of their benefits with respect to changes in the enforcement level. Understanding this along with the size of each groups' stakes in the contest, we see that enforcement may move in the opposite directions of lobbying efforts, in part reflecting sensitivity to and tolerance of corruption. Under certain conditions, sensitivity to corruption gives rise to enforcement levels that benefit the poor. With this, we show the possibility of a poverty trap. 


\section{References}

Andreoni James B., Brian Erard and Jonathan Feinstein. "Tax Compliance," Journal of Economic Literature 36(2) (1998): 818-60.

Das-Gupta, Arindam. "The Economic Theory of Tax Compliance with Special Reference to Tax Compliance Costs," in A. Bagchi (ed.), Readings in Public Finance. New Delhi: Oxford University Press (2005).

Das-Gupta, Arindam and Ira N. Gang. "Decomposing Revenue Effects of Tax Evasion and Tax Structure Changes," International Tax and Public Finance 7(2) (2000): 177-94.

Das-Gupta, Arindam, Gemma B. Estrada, and Donghyun Park. "Measuring Tax Administration Effectiveness and its Impact on Tax Revenue," Nanyang Technological University, School of Humanities and Social Sciences, Economic Growth Centre No. 1601 (2016).

Das-Gupta, Arindam, Shanto Ghosh, and Dilip Mookherjee. "Tax Administration Reform and Taxpayer Compliance in India," International Tax and Public Finance 11(5) (2004): 575-600.

Epstein, Gil S. and Ira N. Gang. "Why Pay Taxes When No One Else Does?," Review of Development Economics 14(2) (2010): 374-385.

Epstein, Gil S. and Shmuel Nitzan. "The Endogenous Determination of Minimum Wage," IZA Working Paper No. 73 (1999).

Epstein, Gil S. and Shmuel Nitzan. "Political Culture and Monopoly Price Determination," Social Choice and Welfare 21(1) (2003): 1-19.

Epstein, Gil S. and Shmuel Nitzan. Endogenous Public Policy and Contests. Springer (2007).

Flatters, Frank and W. Bentley MacLeod. "Administrative Corruption and Taxation," International Tax and Public Finance 2(3) (1995): 397-417.

Myles, Gareth D. and Robin A. Naylor. "A Model of Tax Evasion with Group Conformity and Social Customs," European Journal of Political Economy 12(1) (1996): 49-66.

Schneider, Patrick and Gautam Bose. "Organizational Cultures of Corruption," Journal of Public Economic Theory 19(1) (2017): 59-80. doi:10.1111/jpet.12174

Tullock, Gordon. "Efficient rent-seeking”. In: Buchanan, James M., Robert D. Tollison, and Gordon Tullock. Toward a theory of the rent-seeking society, University Press, Texas (1980): 97-112.

Yitzhaki, Shlomo. "Income Tax Evasion: A Theoretical Analysis," Journal of Public Economics 1(3-4) (1974): 201-02. 